\title{
. Studying Heavy-ion Collisions with Coverage Near Zero Degrees using FAUST-QTS
}

\author{
Paul Cammarata ${ }^{\mathrm{a}, \mathrm{b}, 1, *}$, Matthew B. Chapman ${ }^{\mathrm{a}, \mathrm{c}}$, Alan B. McIntosh ${ }^{\mathrm{a}}$, George \\ A. Souliotis ${ }^{\mathrm{a}, \mathrm{d}}$, Layla Bakhtiari ${ }^{\mathrm{a}, \mathrm{c}}$, Spencer Behling ${ }^{\mathrm{a}, \mathrm{b}}$, Giacomo Bonasera ${ }^{\mathrm{a}, \mathrm{c}}$, \\ Lauren A. Heilborn ${ }^{\mathrm{a}, \mathrm{b}}$, Justin Mabiala ${ }^{\mathrm{a}}$, Larry W. May ${ }^{\mathrm{a}, \mathrm{b}}$, Andrew Raphelt ${ }^{\mathrm{a}, \mathrm{b}}$, \\ Michael D. Youngs ${ }^{\mathrm{a}}$, Andrew Zarrella ${ }^{\mathrm{a}, \mathrm{b}}$, Sherry J. Yennello ${ }^{\mathrm{a}, \mathrm{b}, * *}$ \\ ${ }^{a}$ Cyclotron Institute, Texas A $83 M$ University, College Station, Texas 77843, USA \\ ${ }^{b}$ Department of Chemistry, Texas A $6 M$ University, College Station, Texas 77843, USA \\ ${ }^{c}$ Department of Physics, Texas A 63 M University, College Station, Texas 77843, USA \\ ${ }^{d}$ Laboratory of Physical Chemistry, Department of Chemistry, National and Kapodistrian \\ University of Athens and Hellenic Institute of Nuclear Physics, Athens 15771, Greece
}

12

\section{Abstract}

Heavy-ion collisions around the Fermi energy provide a rich environment for investigating reaction dynamics and provide an opportunity to explore the transition from quasi-fission to multi-fragmentation. A new detection system, FAUST-QTS has been commissioned at Texas A\&M University in order to investigate the reaction dynamics in this transitional energy regime. FAUST-QTS is constructed through the coupling of the FAUST array to a large bore quadrupole triplet spectrometer, and designed to detect heavy fragments near the beam axis coincident with lighter particles.

Keywords: projectile-like fragment, spectrometer, FAUST, transport, time-of-flight

PACS: 29.30.Aj, 25.70-z, 25.70.Mn

\section{Introduction}

Heavy-ion collisions below the Fermi energy (10 - $15 \mathrm{MeV} /$ nucleon) provide a rich environment to study reaction dynamics, as there are a number of competing reactions mechanisms at play[1,2]. This competition between dynamical emission, pre-equilibrium emission and statistical decay of reaction products results in the entanglement of a number of underlying processes. This energy range also provides an opportunity to explore the transition from quasi-fission to multi-fragmentation. Recent theory[3-7] has suggested interesting reaction dynamics via deformations of the reaction system and few body breaking of the deformed, primary reaction partners.

In order to reconstruct the reaction plane and the fragmenting quasi-projectile, the ability to detect the lighter reaction products with high granularity and good solid angle coverage, combined with the detection and identification of the heaviest fragment is required. For systems using inverse kinematics, a forward array

\footnotetext{
Preprint submitted to Nuclear Instruments and Methods A

April 19, 2015

${ }^{*}$ Corresponding author

** Principal corresponding author

Email addresses: pcammarata@comp.tamu.edu (Paul Cammarata), yennello@comp.tamu.edu (Sherry J. Yennello) 77541

${ }^{1}$ Current address: Analytical Technology Center, Dow Chemical Company, Freeport, TX,
}

(c) 2015. This manuscript version is made available under the Elsevier user license

http://www.elsevier.com/open-access/userlicense/1.0/ 
with good coverage near the beam axis is also an important feature. These criteria also hold true for the study of fusion reactions, in inverse kinematics, where the fusion residue lies near the beam axis with lighter particles emitted transverse to the beam axis.

Recently, we have completed construction and commissioning of a new detection system. This system combines the Forward Array Using Silicon Technology (FAUST)[8] with a Quadrupole Triplet Spectrometer (QTS) to form FAUST-QTS. This detector configuration provides good granularity, solid angle coverage, and isotopic identification of particles emitted off beam axis while allowing for the coincident identification of heavy, projectile-like fragment near the beam axis. This manuscript will detail the design and implementation of the FAUST-QTS detection system, simulations to describe the transport of heavy fragments near the beam axis, and initial performance results. Data collected for this initial commissioning campaign of experiments were from reactions of ${ }^{136} \mathrm{Xe},{ }^{124} \mathrm{Sn}+{ }^{64} \mathrm{Ni}$ and ${ }^{124} \mathrm{Xe}+{ }^{58} \mathrm{Ni}$ at $15 \mathrm{MeV} /$ nucleon using the K500 superconducting cyclotron at Texas A\&M University.

\section{Experimental Design}

\subsection{FAUST Upgrade}

The FAUST array[8-11] has been coupled to a large quadrupole triplet spectrometer (QTS). The QTS has been previously used for radioactive-ion beam (RIB)/facility upgrade experiments with BIGSOL[12-16]. FAUST-QTS was designed to make Time-of-Flight (ToF) mass measurements in FAUST and the QTS for heavy projectile-like fragments (PLFs) as well as $\Delta \mathrm{E}-\mathrm{E}$ measurements in FAUST for good isotopic resolution of light charged particles (LCPs) and intermediate mass fragments (IMFs) $[8,17]$. FAUST is constructed of 68 Si$\mathrm{CsI}(\mathrm{Tl}) \Delta \mathrm{E}-\mathrm{E}$ telescopes arranged into five concentric square rings of detectors, as can be seen in Figure 1. The rings of FAUST are designed as squares projected spheres. The array provides $89.7 \%$ solid angle coverage from $2.3^{\circ}$ to $33.6^{\circ}$. Partial coverage exists from $1.6^{\circ}$ to $2.3^{\circ}$ and from $33.6^{\circ}$ to $45^{\circ}$. Each $\mathrm{Si}$ detector in FAUST is a $2 \mathrm{~cm} \mathrm{x} 2 \mathrm{~cm}$ single edge mounted, $300 \mu \mathrm{m}$ thick silicon wafer backed by a $\mathrm{CsI}(\mathrm{Tl})$ inorganic scintilator read out by photodiode.

The ToF mass identification in FAUST is achieved via newly designed integrated charge sensitive, timing pickoff (CS/TPO) pre-amplifiers as part of the upgrade to FAUST. The external Zepto systems preamplifiers[18] were replaced with new charge-sensitive timing pick-off (CS-TPO) preamplifiers and motherboards from RIS-Corp[19] for Rings A-D silicon detectors. The new CS-TPO preamplifiers occupy a slightly larger footprint than the Zepto systems preamplifiers, as seen in Figure 2, due to two extra pin connectors for the timing signal.

The timing on the new CS-TPO preamplifiers is accomplished by detecting charge fluctuations at the detector input to the charge sensitive portion of the CS-TPO. Specifically, the change in JFET current is passed through the emitter of the pnp transistor (connected to the JFET source/emitter) and is amplified 


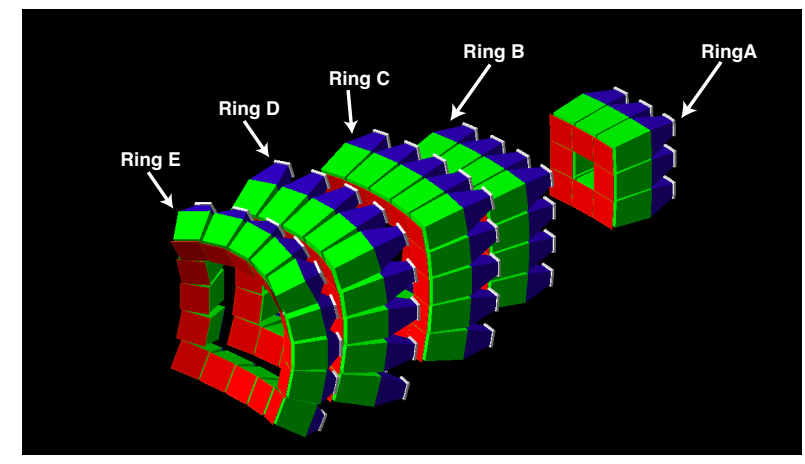

Figure 1: A 3D rendering of the FAUST array from the GEANT4 environment.

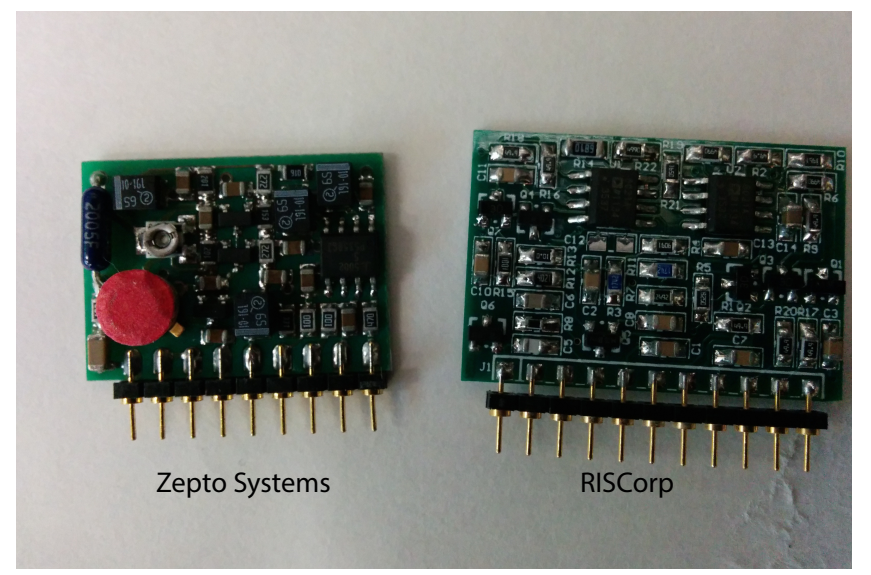

Figure 2: Zepto Systems preamplifier (left) and RIS-Corp[19] preamplifier (right) shown sideby-side.

73 to provide a timing signal in the amplifier circuit shown in Figure 3. There is 74 a sharp change in JFET current for the time it takes to collect the charge de75 posited by the particle of interest in the detector. This change in current (flux) 76 generates the timing signal. This signal is then integrated to generate a voltage 77 signal with $\sim 1 \mathrm{~ns}$ rise time. This allows for fast-timing of the incident particle 78 simultaneously with charge sensitive pre-amplification in one integrated circuit 79 chip-set without added bulk or additional cabling. The new motherboards are o backwards compatible with the Zepto systems pre-amplifiers and allow for in1 dividual biasing of each Si detector via WIENER Plein and Baus MPOD High 2 Voltage power supply modules.

In order to generate a common stop timing signal, a thin film, fast plastic scintillator (BC-408) was installed up-stream of FAUST. The plastic scintillator was sandwiched and glued between two Lucite light guides and then wrapped with $2.4 \mu \mathrm{m}$ aluminized Mylar. The light guide assembly was then mounted 


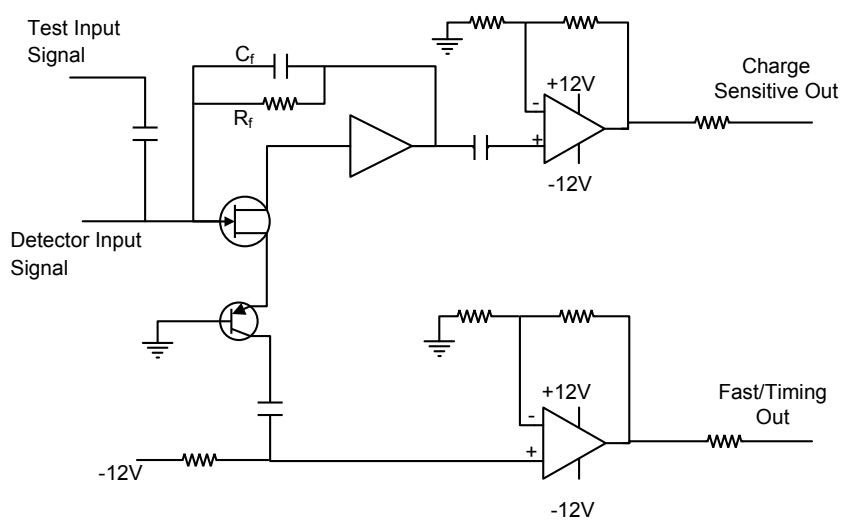

Figure 3: Brief schematic of the RIS-Corp[19] charge sensitive, timing pick-off preamplifiers.

within a custom designed housing. The light generated from the BC408 plastic was read out via a Hammamatsu R1635 photomultiplier tube. The fast signal from the photomultiplier tube was used as the common stop in the timing electronics after being processed by a Tennelec 454 constant fraction discriminator (CFD) and subsequently delayed by 135ns. The timing signal from each of the $68 \mathrm{Si}$ detectors in FAUST was processed by CAEN V812B CFDs and used as the start timing signal. The time difference between the CFD signals from the FAUST silicon detectors (start) and the fast plastic (stop) was determined via CAEN V775 time-to-digital converter set to maximum resolution (35 ps/channel, $143 \mathrm{~ns}$ full range). The preamplified energy signals from both the $\mathrm{Si}$ and $\mathrm{CsI}(\mathrm{Tl})$ detectors in FAUST were shaped by custom bipolar shaping amplifiers, as described by Ref [8], and read out by Mesytec MADC32 analogto-digital converters.

In offline testing, we were able to achieve a time resolution of up to $\sim 150$ ps full-width half-max (FWHM), as seen in Figure 4. This ideal resolution was possible because of a small angular coverage of the detector due, largely, to distance from the source, low noise and single isotope analysis using elastically scattered ${ }^{129} \mathrm{Xe}+{ }^{197} \mathrm{Au}$ at $15 \mathrm{~A} \mathrm{MeV}$. However, in the experiment, we were able to achieve a maximum timing resolution of $\sim 400$ ps. This was highly dependent on the condition/level of radiation damage, background noise, large angular coverage of each telescope $\left(\theta_{\text {lab }} \sim 8^{\circ}\right)$ and a wider range of isotopes detected at varying energies on each individual silicon detector. On average, most silicon detectors had a timing resolution between 600-1200ps during the actual experiment.

\subsection{Quadrupole Triplet Spectrometer}

The Quadrupole Triplet Spectrometer (QTS) consists of three large bore (8 inch inner-diameter) quadrupole magnets focusing in the $\mathrm{X}-$, $\mathrm{Y}-$, and then 


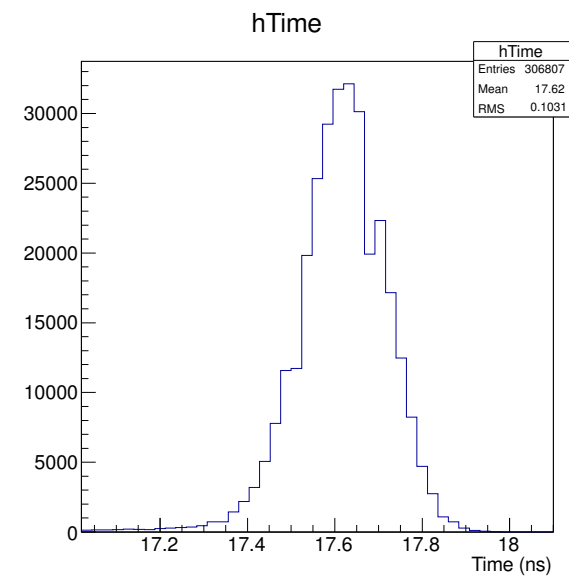

Figure 4: Preamplifier resolution in a best case scenario in the lab using a plastic scintillator and FAUST silicon detectors. Maximum resolution is on the order of $150 \mathrm{ps}$ at full-width half-maximum.

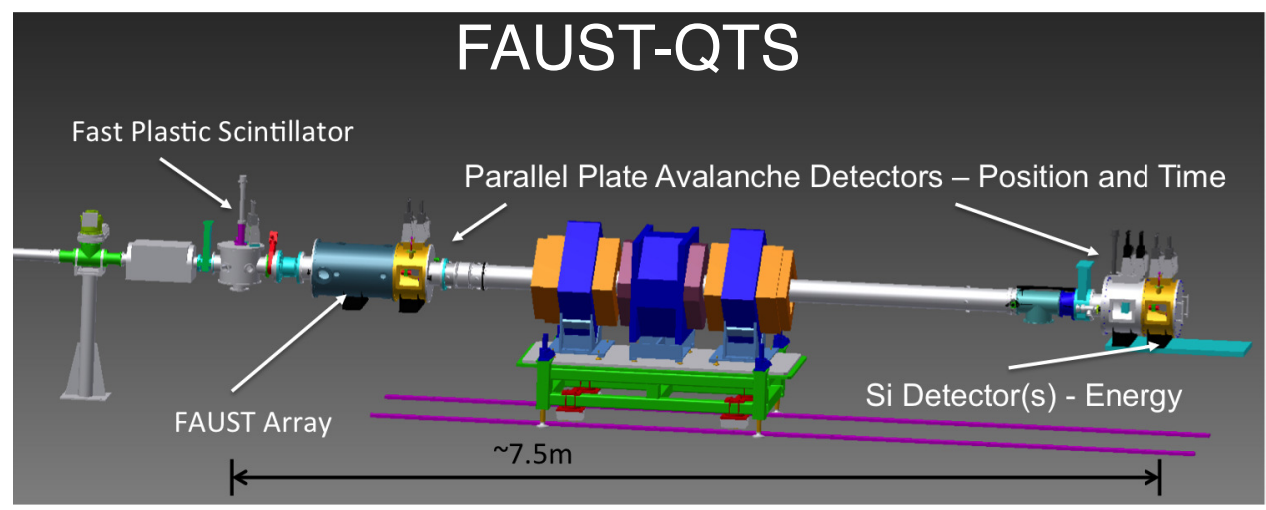

Figure 5: A 3-dimensional rendering of the FAUST-QTS beam line showing detector and beam-line component positioning.

$\mathrm{X}$-direction (respectively) relative to the beam axis. The spectrometer also consists of two customizable detection chambers, one before and one after the magnets, to allow ToF mass particle identification (PID) and/or $\Delta \mathrm{E}-\mathrm{E}$ isotopic identification depending on the energy range of the particles of interest. For this experiment we employed ToF mass PID.

The ToF mass measurements in the QTS were made using Parallel Plate Avalanche Counters (PPACs) [20] to measure the fragment time-of-flight coupled with a $1000 \mu \mathrm{m}$ silicon detector (MSQ-25, Micron Semiconductor, Lancing, UK) at $\theta_{\text {lab }}=0^{\circ}$ for accurate particle energy measurement. The PPACs were mounted before and after the quadrupole triplet, for accurate time of flight measurement. The $5 \mathrm{~cm} \times 5 \mathrm{~cm}, 1000 \mu \mathrm{m}$ thick, quadrant segmented silicon detector was 
mounted after the last PPAC for accurate particle energy measurement and was chilled to $5^{\circ} \mathrm{C}$ with thermal and vibrational isolation. A $1 / 4$ inch thick aluminum collimator was placed just upstream of the $1000 \mu \mathrm{m}$ silicon detector in order to decrease the rate of elastically scattered beam particles not stopped by a Faraday cup (covering $\theta_{\text {lab }}=0^{\circ}-2.3^{\circ}$ ) mounted between FAUST and PPAC\#1. The full detector arrangement is shown in Figure 5 via 3D rendered CAD drawing of the FAUST-QTS beam line.

As has been previously mentioned and shown in Figure 5, the PPACs were mounted one in front of the triplet and one after the triplet spectrometer but before the silicon detector. Each PPAC, with 6 torr of isobutane gas flowing at $30-40 \mathrm{cc} /$ second, was biased to $530 \mathrm{~V}$ via a Tennelec High Voltage Power Supply. The signals from the up (U), down (D), left (L) and right (R) signal were pre-amplified by custom designed hex-amplifiers. The U, D, L and R signals were then shaped by a CAEN N568B, 16-channel Spectroscopy amplifier. The resultant amplified signals were then digitized via Mesytec MADC32 peak sensing ADCs. The timing signal from the PPAC was passively split via Spieler Timing Pick-off amplifier (LBL-21X7422)[21, 22], with half of the signal being pre-amplified, shaped, and digitized like the U, D, L and R signals. The other half of the passively split signal generated a fast timing signal that was cleanedup and amplified by an ORTEC timing filter amplifier and discriminated by a Tennelec 454 CFD. The signals from both PPACs were treated in the same way. The timing signals resulting from both PPACs were then routed from the CFDs and measured via an ORTEC 566 time-to-amplitude converter (TAC) with PPAC\#2 utilized as the start signal and PPAC\#1 as the stop (effectively in common stop mode). The TAC output was then digitized via Mesytec MADC32 ADC.

Optimal magnetic settings for the QTS were determined through a series of optical transport calculations. Basic, $1^{\text {st }}$ order optics were calculated analytically using the TRANSPORT[23] code based on the flight path of the beam through FAUST-QTS to the detection plane at the $5 \mathrm{~cm} \times 5 \mathrm{~cm}$ Si detector located at the end of the QTS. This allowed for the determination of the base magnetic field settings (in kiloGauss) for the spectrometer and was the basis for higher-order calculations via RAYTRACE[24] and COSY-Infinity[25]. Magnet current settings for experimental data collection were determined by using the measured minimum current to generate a magnetic field as measured by a Hall probe. These currents were then compared to previous campaigns of experimental data[12-16] taken with the QTS. A linear fit for current(A) as a function of magnetic rigidity $(\mathrm{B} \rho)$ was obtained.

\section{Quadrupole Triplet Transport Simulations}

An important consideration of using a spectrometric device is understanding the physics that affect particle transport. This is important in order to anticipate what particles are likely to be transported and detected, in addition to determining which particles can be separated spectroscopically. The particles' mass, charge state, angle of entrance into the spectrometer, and momentum are 
all important considerations that must be carefully taken into account when calculating charged particle transport flight paths.

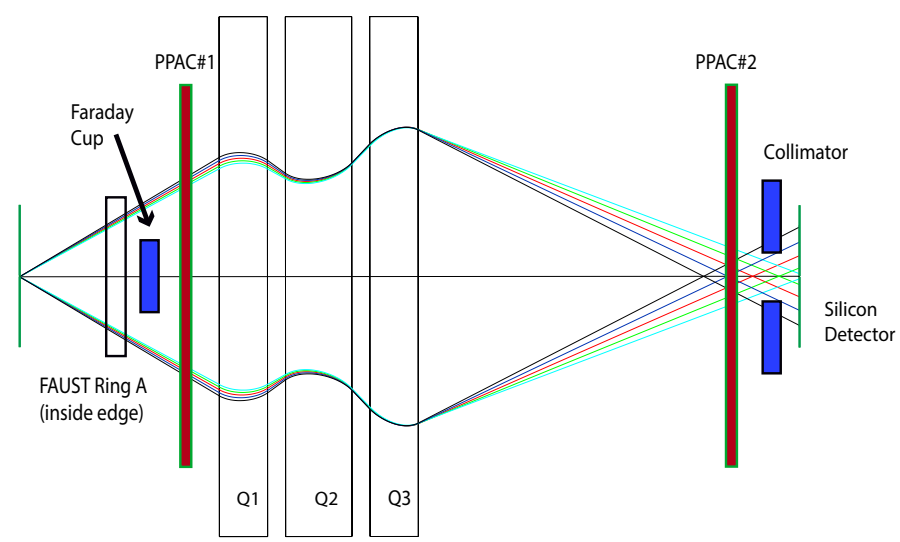

(a) XZ-optics coordinate plane

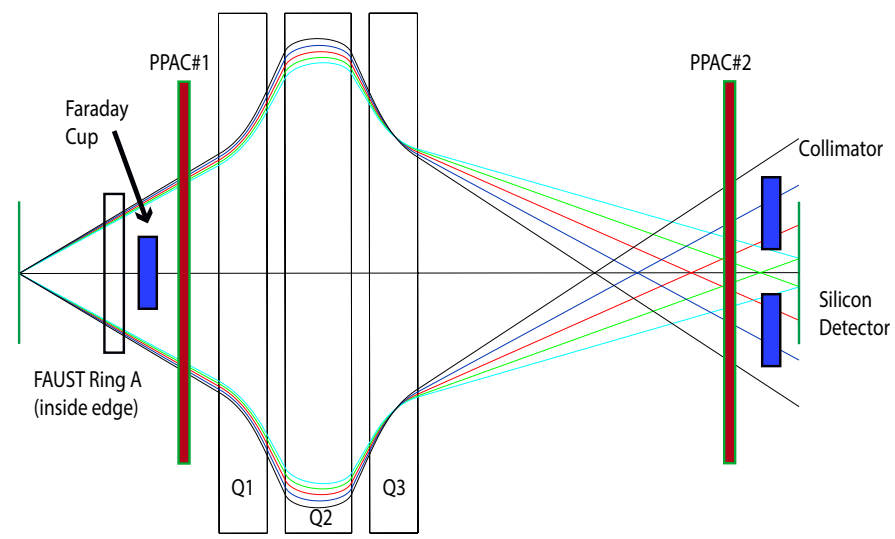

(b) YZ-optics coodrinate plane

Figure 6: COSY-Infinity simulations showing the XZ- and YZ-plane flight path of a PLF emitted at $\theta_{\mathrm{lab}}=1.5^{\circ}$. The five, discrete magnetic rigidities are centered around a $\mathrm{B} \rho=1.45 \mathrm{Tm}$ for the QTS magnets. Red $= \pm 0 \% \mathrm{~B} \rho$, Light Blue $=+5 \% \mathrm{~B} \rho$, Dark Blue $=-5 \% \mathrm{~B} \rho$, Green $=+10 \% \mathrm{~B} \rho$, and Black $=-10 \% \mathrm{~B} \rho$

Simulations have been examined for a number of probable cases of transport through the QTS using RAYTRACE and COSY-Infinity. This was done in order to examine the theoretical, optical transport considerations required to conduct the proposed experiment. This is especially important since the entrance flight path is significantly shorter than the exit flight path. This, combined with the spectrometer being positioned at zero degrees with respect to the beam, requires careful calculation of the magnetic fields required to transport particles of interest. 
Simulations via COSY-Infinity illustrate the effects of the $\mathrm{B} \rho$ on the ability to focus particles through the triplet, as shown in Figure 6. Figure 6 illustrates several values of $\mathrm{B} \rho$ for a PLF with a single theta value of $\theta_{\mathrm{lab}}=1.5^{\circ}$. As the value of theta increases (up to a maximum of $\theta_{\text {lab }}=2.3^{\circ}$ ) so do the aberrations in the focal image. This is particularly true for the YZ-plane where it can be seen that even at $\theta_{\text {lab }}=1.5^{\circ}$ the flight paths of the particles are nearing the edge of the triplet aperture in the $2^{\text {nd }}$ quadrupole magnet (Q2). The aberration in the XZ-plane is less than that of the YZ-plane simply because Q1 focuses initially in the X-direction and, thereby, defocuses in the Y-direction. Some adjustment can be made to help mitigate some of the higher-order effects by decoupling the magnetic fields on Quardruple\#1 (Q1) and Quadrupole\#3 (Q3), both X-focusing quadrupoles. By decreasing the Q3 magnetic field by $5 \%$ of that on Q1, there is a significant decrease in the predicted aberrations. Typically, for a symmetric spectrometer, the coupling of the X-focusing magnets (Q1 and Q3) would be preferred. However, the decoupling of Q1 and Q3 in the QTS improves the focusing power by decreasing the focal plane aberration and allows for fine, empirical adjustment when tuning the fragments of interest.

In addition to aberrations in the focal plane image, and exclusion of reaction products not of interest, it is also important to ensure that scattered, beamlike particles are not overwhelming the data stream or masking the particles of interest detected at the focal plane of the QTS. To address this concern, GEANT4[26] was used to simulate elastically scattered beam particles through FAUST-QTS. The entire FAUST array and the QTS, up to the entrance of the first quadrupole magnet, were constructed inside of GEANT4. The simulation included the Faraday cup (beam block) located between FAUST and the QTS at zero degrees, and can be visualized in Figure 7. The physical beam pipe

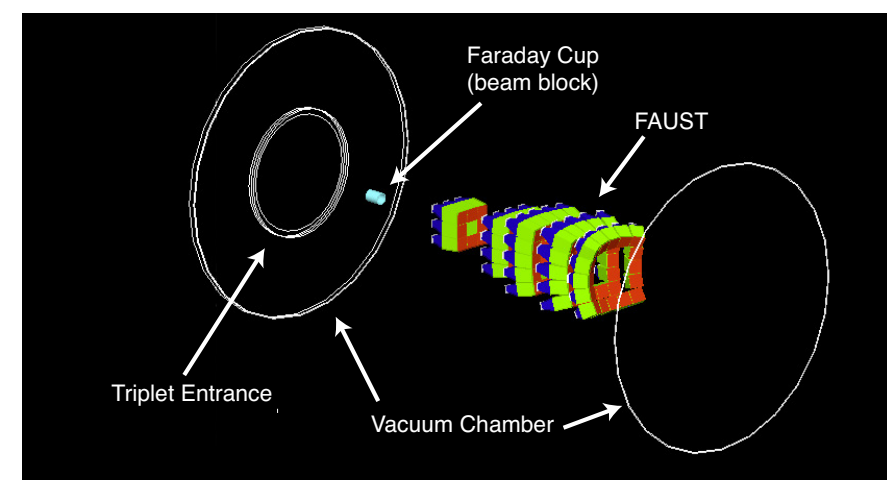

Figure 7: Cross Sectional view of the FAUST-QTS in GEANT4. The FAUST array is shown as the array of five, multicolored rings. The beam pipe is hidden to allow the display of the detectors and beam line components. The small blue cylinder behind the FAUST array is the Faraday Cup covering a range of $\theta_{\text {lab }}=0-0.9^{\circ}$.

is not shown in Figure 7 for display purposes. However, it was included in the simulation for geometric constraints. The elastic scattering cross-section 
as a function of $\theta_{\text {lab }}$ was extracted from LISE $++[27,28]$ using the reaction of ${ }^{136} \mathrm{Xe}+{ }^{64} \mathrm{Ni}$ at $15 \mathrm{MeV} /$ nucleon.

One billion primary elastic events were simulated covering an angular range that encompassed particles transmitted through the center of FAUST $\left(\theta_{\text {lab }}<\right.$ $2.3^{\circ}$ ). Ring A of FAUST was allowed to act as a collimator in order to achieve the correct, square angular distribution in $\theta_{\text {lab }}$ and $\phi_{\text {lab }}$. Of particular interest were particles that interacted with the knife edge of the cup [29, 30] thereby losing an appreciable amount of energy, thus possibly resembling particles of interest from projectile-target reactions. Once the elastically scattered particles interacted with the edge of the cup, it was postulated that a small number of particles could lose energy, be inelastically scattered, enter the triplet, and be mistaken for particles of interest at the focal plane.

The knife-edge scattered particles span a range of energy that could overlap with the expected energy range of the PLFs of interest in this type of experiment. This, combined with the fact that the inelastic (knife-edge) scattered beam particles are near the expected mass of a number of the PLFs of interest, drew some concern. However, low beam rate $\left(\sim 1.5 \times 10^{5}\right.$ particles per second) and theoretical spectrometer transport efficiencies near $68 \%$ for PLFs with a $\mathrm{B} \rho^{\text {particle }} \leq 90 \% \mathrm{~B} \rho^{\text {beam }}$ resulted in a negligible probability of detecting a coincident IMF in FAUST with a scattered beam particle in the QTS. This probability rapidly diminishes for particles of interest where $\mathrm{B} \rho^{\text {particle }}<<\mathrm{B} \rho^{\text {beam }}$ particularly in the case where the particle to be detected in the QTS is a fusionlike residue and is far away from beam rigidity.

\section{Experimental Performance}

PLF-like particles were transported through the triplet spectrometer utilizing similar spectrometer settings as described above via the optical transport simulations. The focal PPACs, as expected, have inherently good position resolution. This is exemplified in Figure 8a where it illustrates the position resolution of the PLF-like particles focused through the QTS. In Figure 8b, the physical mask constructed of 0.036" thick brass with the Texas A\&M Logo CNC milled into the face of the mask. After the particles were focused by the QTS and passed through PPAC\#2, they were then filtered by the logo mask before being detected by the silicon detector at the focal plane. The logo mask was mounted $3 \mathrm{~cm}$ upstream of the silicon detector on a retractable arm.

The goal of pairing detection devices in the FAUST-QTS system is to provide a coincident measurement of heavier residues near zero degrees (off beam axis) and lighter charged particles emitted across a wider angular range. The isotopic resolution of FAUST remains unchanged from its original capabilities with isotopic resolution beyond $\mathrm{Z}=9$. Unit mass resolution may be possible in the QTS, at higher bombarding energies, with the planned upgrade to a $\Delta \mathrm{E}$ E technique at the QTS focal plane. However, the \pm 3 mass unit resolution achieved in this commissioning campaign will be sufficient to extract information about the dynamical nature of the reaction in this energy regime. The mass measurement of the PLFs made via ToF in the QTS, combined with the 


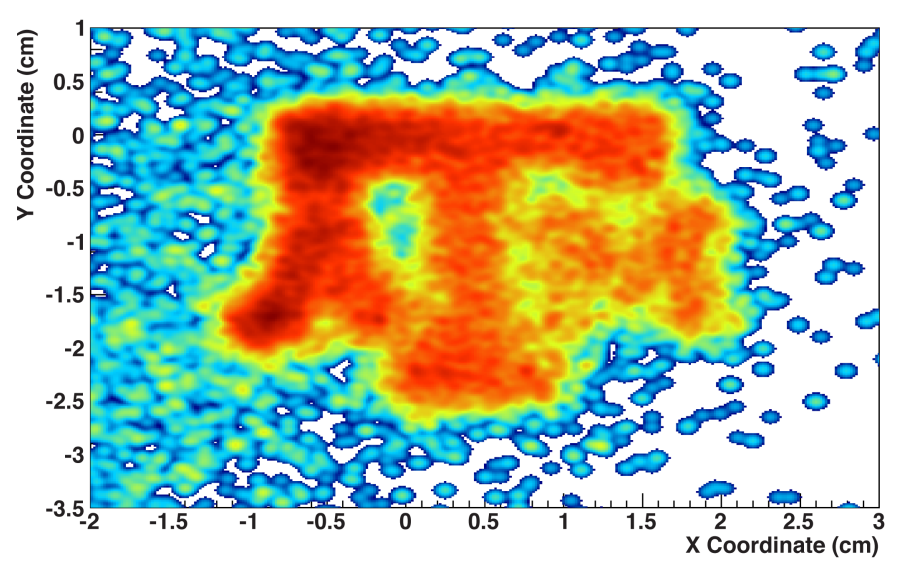

(a) Texas A\&M Logo as seen on PPAC\#2 for PLF-like events. The PLF like events were coincident with a silicon trigger after passing through both PPAC\#2 and the Texas A\&M Logo mask.

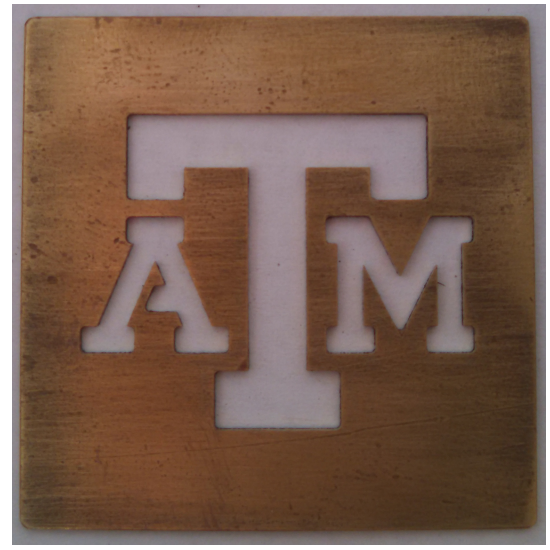

(b) Texas A\&M Logo Mask. The mask was placed between PPAC\#2 (downstream) and QTS silicon semiconductor detector (as shown in Figure 5)

Figure 8: Experimental data showing PLF-like particles focused by the QTS, though PPAC\#2, a mask utilizing the Texas A\&M University logo and detected in coincidence with a silicon detector at the focal plane of the QTS.

information gained via the isotopically resolved IMFs in FAUST, will be sufficient to explore dynamical phenomena associated with size asymmetry, relative energies, and angular correlations. Additionally, this will allow for the ability to trace the evolution of isospin and its interplay with dynamical phenomena, dominant at $15 \mathrm{MeV} /$ nucleon. 


\section{Summary}

A new beam line and detection system has been built for the study of heavyion reaction products below the Fermi energy. The FAUST-QTS offers the ability to study heavy reaction products near the beam axis via a zero-degree, quadrupole spectrometer combined with a forward array to measure the off beam axis LCPs and IMFs. The detector arrangement has met the design criteria and has been recently used to study the reaction dynamics of ${ }^{136} \mathrm{Xe},{ }^{124} \mathrm{Sn}+{ }^{64} \mathrm{Ni}$ and ${ }^{124} \mathrm{Xe}+{ }^{58} \mathrm{Ni}$ at $15 \mathrm{MeV} /$ nucleon. Work is currently underway to improve the energy and time (and thereby the mass) resolution of the QTS. FAUST currently offers good isotopic resolution via $\Delta E-E$ just as it has in the past. Further experiments are underway to utilize FAUST-QTS for temperature and particle correlation experiments.

\section{Acknowledgments}

We would like to thank the staff of the Texas A\&M University Cyclotron Institute for their help in commissioning the experimental setup and for the excellent beam quality delivered on target. We would also like to thank the Texas A\&M University Chemistry Department's machine shop for going above and beyond in fabricating critical beam-line components for this experiment. We are also grateful to the Texas A\&M Super-Computing facility and the Texas A\&M Chemistry Department's Laboratory for Molecular Simulations for the generous grant of over 10 million High Performance Computing (HPC) processor hours (combined). This material is based upon work supported by the U.S. Department of Energy, Office of Science, Office of Nuclear Physics under Award Number DE-FG03-93ER40773, Robert A. Welch Grant Number A-1266 and NSF Grant Numbers CHE-0541587 and PHY-1102511.

[1] De Filippo, E. and Pagano, A., Eur. Phys. J. A 50 (2014) 32.

[2] Kohley, Z. and Yennello, S.J., Eur. Phys. J. A 50 (2014) 31.

[3] Colonna, M., J. Phys. Conf. Ser. 168 (2009) 012006.

[4] Rizzo, C., Baran, V., Colonna, M., Corsi, A. and Di Toro, M., Phys. Rev. C 83 (2011) 014604.

[5] Di Toro, M., Baran, V., Colonna, M., Ferini, G., Gaitanos, T. et al, Nucl. Phys. A 787 (2007) 585.

[6] Shvedov, L., Colonna, M. and Di Toro, M., Phys. Rev. C 81 (2010) 054605.

[7] Giuliani, G., Zheng, H. and Bonasera, A., Prog. Part. Nucl. Phys. 76 (2014) 116.

[8] Gimeno-Nogues, F., Rowland, D., Ramakrishnan, E., Ferro, S., Vasal, S. et al, Nucl. Instruments Methods Phys. A 399 (1997) 94. 
[9] Rowland, D.J., A Study of the Projectile Fragmentation of Isobaric Nuclei, $\mathrm{PhD}$ thesis, Texas A\&M University, 2000.

[10] Keksis, A., N/Z Equilibration in Deep Inelastic Collisions and the Fragmentation of the Resulting Quasiprojectiles, PhD thesis, Texas A\&M University, 2007.

[11] Soisson, S.N., Isospin Dependence of Fragmentation, PhD thesis, Texas A\&M University, 2010.

[12] Souliotis, G.A., Shetty, D., Keksis, A., Bell, E., Jandel, M. et al, Phys. Rev. C 73 (2006) 024606.

[13] Souliotis, G.A., Botvina, A.S., Shetty, D.V., Keksis, A.L., Jandel, M. et al, Phys. Rev. C 75 (2007) 011601.

[14] Souliotis, G.A., Keksis, A., Stein, B., Veselsky, M., Jandel, M. et al, Nucl. Instruments Methods Phys. B 261 (2007) 1094.

[15] Barbui, M., Pesente, S., Nebbia, G., Fabris, D., Lunardon, M. et al, Nucl. Instruments Methods Phys. B 265 (2007) 605.

[16] Souliotis, G.A., Stein, B., Veselsky, M., Galanopoulos, S., Keksis, A. et al, Nucl. Instruments Methods Phys. B 266 (2008) 4692.

[17] Stein, B.C., Soisson, S.N., May, L.W., Dienhoffer, R.Q., Souliotis, G.A. et al, AIP Conf. Proc. 700 (2009) 700.

[18] Davin, B., de Souza, R.T., Yanez, R., Larochelle, Y., Alfaro, R. et al, Nucl. Instruments Methods Phys. A 473 (2001) 302.

[19] Todd, R., RIS-Corp, 5905 Weisbrook Lane, Suite 102 Knoxville, TN 37909, 2013.

[20] Swan, D., Yurkon, J. and Morrissey, D.J., Nucl. Instruments Methods Phys. A 348 (1994) 314.

[21] Spieler, H., Untersuchung des Zwei-Neutron-Transfers an den Reacktionen $120 \mathrm{Sn}+18 \mathrm{O}$ und $122 \mathrm{Sn}+16 \mathrm{O}, \mathrm{PhD}$ thesis, Technische Hochschule Munchen, 1974.

[22] Spieler, H., Körner, H. and Rehm, K., Zeitschrift für Phys. A Hadron. Nucl. 250 (1976) 241.

[23] Brown, K. and Carey, D., SLAC-91, Rev. 3 (1983).

[24] Kowalski, S. and Enge, H., Nucl. Instruments Methods Phys. A 258 (1987) 407.

[25] Makino, K. and Berz, M., Nucl. Instruments Methods Phys. A 558 (2006) 346. 
329 [26] Agostinelli, S., Allison, J., Amako, K., Apostolakis, J., Araujo, H. et al, $330 \quad$ Nucl. Instruments Methods Phys. A 506 (2003) 250.

331 [27] Tarasov, O. and Bazin, D., Nucl. Phys. A 746 (2004) 411.

332 [28] LISE ++ (http://lise.nscl.msu.edu/lise.html).

333 [29] Courant, E.D., Rev. Sci. Instrum. 22 (1951) 1003.

${ }_{334}$ [30] Burge, E.J. and Smith, D.A., Rev. Sci. Instrum. 33 (1962) 1371. 